\title{
SIMULATION OF CONSTRAINED ELASTIC CURVES AND APPLICATION TO A CONICAL SHEET INDENTATION PROBLEM
}

\author{
SÖREN BARTELS
}

\begin{abstract}
We consider variational problems that model the bending behavior of curves that are constrained to belong to given hypersurfaces. Finite element discretizations of corresponding functionals are justified rigorously via $\Gamma$-convergence. The stability of semi-implicit discretizations of gradient flows is investigated which provide a practical method to determine stationary configurations. A particular application of the considered models arises in the description of conical sheet deformations.
\end{abstract}

\section{DEDiCATED TO THE MEMORY OF JOHN W. BARRETT}

\section{INTRODUCTION}

The elastic flow of curves has attracted considerable attention among applied and numerical analysts within the last decades, cf., e.g., LS85; DKS02; DLP14] for analytical results, and [DDE05, BGN08; DD09; BGN10; BGN11; BGN12; Bar13, PS17; BRR18; BGN19] for results concerning the discretization. Corresponding applications occur in the modeling of phase transitions, the description of large deformations of elastic rods and ribbons AP10, and prediction of prefered shapes of molecules [CGM06; CS00]. For the class of inextensible curves, which arise naturally as dimensionally reduced descriptions in nonlinear elasticity [Ant05: MM03], recent developments concerning the numerical treatment of partial differential equations with holonomic constraints such as harmonic maps turned out be useful for their efficient approximation, cf. Bar05; Bar+07; Bar16]. In this article we consider curves that are restricted to belong to given surfaces and whose behavior is determined by appropriate bending energies. To model their relaxation dynamics and find stationary configurations of low energy we adapt techniques developed in Bar13 to develop convergent finite element discretizations and stable iterative numerical schemes. Our approach provides an alternative to the methods developed in BGN12; BGN19. Here, motivated by applications in nonlinear elasticity, we consider curves in euclidean space that are parametrized by arclength which allows for an efficient numerical treatment. Related analytical contributions are contained in Lin91; Koi96].

Date: April 24, 2020.

2010 Mathematics Subject Classification. 65N12 63N30 74K10.

Key words and phrases. Elasticity, rods, surfaces, discretization, conical sheets. 
1.1. Constrained nonlinear bending. We first consider relaxation processes of curves $u$ on a given surface $S$ whose bending behavior is determined by the functional

$$
I[u]=\frac{1}{2} \int_{0}^{L}\left|u^{\prime \prime}\right|^{2} \mathrm{~d} x .
$$

Here, we require $u:(0, L) \rightarrow \mathbb{R}^{3}$ to be an arclength parametrized curve, i.e., that $\left|u^{\prime}(x)\right|=1$ for all $x \in(0, L)$, so that $\left|u^{\prime \prime}\right|^{2}$ is the squared curvature of the curve parametrized by the function $u$. The constraint

$$
u(x) \in S
$$

for all $x \in(0, L)$ restricts the curve to belong to the regular hypersurface $S \subset \mathbb{R}^{3}$. We also incorporate boundary conditions modeled by a bounded and linear functional $L_{\mathrm{bc}}: H^{2}\left(\Omega ; \mathbb{R}^{3}\right) \rightarrow \mathbb{R}^{\ell}$ and a vector $\ell_{\mathrm{bc}} \in \mathbb{R}^{\ell}$. The setting may describe the behavior of a wire on a magnetic surface neglecting effects related to twist. Corresponding torsion contributions can however be directly included, cf. [BR19]. We thus consider the following constrained minimization problem.

$$
\left(\mathrm{P}_{\text {bend }}\right) \quad\left\{\begin{array}{l}
\text { Find a minimizing curve } u \in H^{2}\left(0, L ; \mathbb{R}^{3}\right) \text { for } \\
I[u]=\frac{1}{2} \int_{0}^{L}\left|u^{\prime \prime}\right|^{2} \mathrm{~d} x \\
\text { subject to } u(x) \in S,\left|u^{\prime}(x)\right|^{2}=1 \text { for all } x \in[0, L] \\
\text { and } L_{\mathrm{bc}}[u]=\ell_{\mathrm{bc}} .
\end{array}\right.
$$

For an initial configuration described by a function $u_{0}$ and for given boundary conditions, e.g., that the wire is clamped at one end, the relaxation of the bending energy is modeled by the formal gradient flow evolution

$$
\partial_{t} u=-I^{\prime}[u]+\left(\lambda u^{\prime}\right)^{\prime}+\mu \Phi_{S}^{\prime}(u)
$$

for a family of curves $(u(t))_{t \in[0, T]}$ satisfying the the initial, holonomic, and boundary conditions

$$
u(0)=u_{0}, \quad\left|u^{\prime}\right|^{2}=1, \quad \Phi_{S}(u)=0, \quad L_{\mathrm{bc}}[u]=\ell_{\mathrm{bc}} .
$$

The functions $\lambda$ and $\mu$ are Lagrange multipliers related to the arclength and surface constraints, respectively, where we assume that the surface $S$ is given as the zero level set of the function $\Phi_{S}$. With the backward difference quotient operator

$$
d_{t} a^{k}=\frac{1}{\tau}\left(a^{k}-a^{k-1}\right)
$$

we use a time-stepping scheme that linearizes the constraints at a previous approximation. By restricting to test functions that belong to the intersection of the kernels of the linearized constraints this eliminates the explicit occurence of the Lagrange multipliers. Since the time-derivative obeys the 
same linear constraints we obtain for an appropriate inner product $(\cdot, \cdot)_{*}$ and the $L^{2}$ inner product $(\cdot, \cdot)$ the time-stepping scheme

$$
\left(d_{t} u^{k}, v\right)_{*}+\left(\left[u^{k}\right]^{\prime \prime}, v^{\prime \prime}\right)=0
$$

subject to the inclusions

$$
d_{t} u^{k}, v \in \mathcal{F}\left[u^{k-1}\right],
$$

where the set $\mathcal{F}\left[u^{k-1}\right]$ contains the linearized constraints, i.e., for a given curve $\widehat{u}$ we have

$$
\mathcal{F}[\widehat{u}]=\left\{v \in H^{2}\left(0, L ; \mathbb{R}^{3}\right): \widehat{u}^{\prime} \cdot v=0, \Phi_{S}^{\prime}(\widehat{u}) \cdot v=0, L_{\mathrm{bc}}[v]=0\right\} .
$$

The time-stepping scheme thus requires solving linearly constrained linear systems of equations, where the constraints are pointwise. We show that the scheme is unconditionally energy decreasing and that the violation of the constraints is controlled by the step size independently of the number of iterations. Our spatial discretization uses an $\mathrm{H}^{2}$-conforming ansatz and imposes the constraints at the nodes of a partitioning of the reference interval $(0, L)$. We justify the spatial discretization by proving its $\Gamma$-convergence to the continuous minimization problem.

1.2. Geodesic curvature. An intrinsic variant of the constrained variational problem arises, e.g., in the description of phase separation processes on surfaces. It replaces the curvature $\kappa=\left|u^{\prime \prime}\right|$ by the geodesic curvature $\kappa_{g}$. For an arclength parametrized curve $u:(0, L) \rightarrow S$ it is defined as

$$
\kappa_{g}^{2}=\left|u^{\prime \prime}\right|^{2}-\left|u^{\prime \prime} \cdot n_{S}(u)\right|^{2}=\left|u^{\prime \prime} \times n_{S}(u)\right|^{2},
$$

where $n_{S}=\Phi_{S}^{\prime} /\left|\Phi_{S}^{\prime}\right|$ is a unit normal field on $S$ and where we used that $u^{\prime \prime} \cdot u^{\prime}=0$. The corresponding energy functional

$$
I[u]=\frac{1}{2} \int_{\Omega} \kappa_{g}^{2} \mathrm{~d} s
$$

still controls the $H^{2}$ norm of $u$ since the normal part of the curvature is bounded by the curvature of $S$, i.e., we have

$$
\left|u^{\prime \prime}\right|^{2} \leq \kappa_{g}^{2}+c_{S}^{2},
$$

where $c_{S}$ is the maximum of the principal curvatures of $S$. This estimate is not availabe when only nodal values of a piecewise polynomial curve $u_{h}$ belong to $S$. To cope with this aspect we introduce a stabilization via a damping parameter $\gamma \leq 1$ in the energy functional.

$$
\left(\mathrm{P}_{\text {geod }}^{\gamma}\right) \quad\left\{\begin{array}{l}
\text { Find a minimizing curve } u \in H^{2}\left(0, L ; \mathbb{R}^{3}\right) \text { for } \\
\quad I_{\gamma}[u]=\frac{1}{2} \int_{0}^{L}\left|u^{\prime \prime}\right|^{2}-\gamma\left|u^{\prime \prime} \cdot n_{S}(u)\right|^{2} \mathrm{~d} x \\
\text { subject to } u(x) \in S,\left|u^{\prime}(x)\right|^{2}=1 \text { for all } x \in[0, L] \\
\text { and } L_{\mathrm{bc}}[u]=\ell_{\mathrm{bc}} .
\end{array}\right.
$$




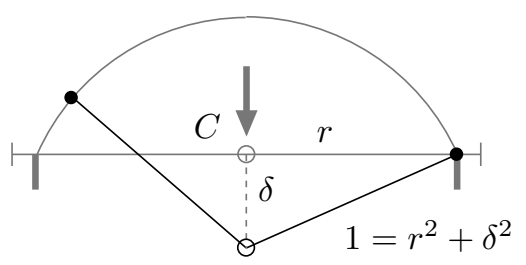

Figure 1. A point $C$ of an initially flat elastic sheet (gray line representing cross section) is displaced by a distance $\delta$. The resulting deformation is constrained by a circular obstacle at distance $r$ to $C$. Points on the deformed sheet (black lines) at distance $\left(r^{2}+\delta^{2}\right)^{1 / 2}$ to the center $C$ either touch the obstacle (right end point) or are above it (left end point).

We prove that the stabilized problems converge in a variational sense to the unstabilized original problem as $\gamma \rightarrow 1$. The stabilization allows us to prove convergence of discretizations. As an alternative to or in combination with stabilizations additional constraints may be imposed to ensure that discrete curves remain sufficiently close to the surface $S$ so that their second derivative in normal direction is controlled by the curvature of the surface. This approach however leads to difficulties in the iterative solution. For the stabilized problem we follow the ideas described above with an explicit treatment of the nonlinear term. Hence, we compute a sequence $\left(u^{k}\right)_{k=0,1, \ldots}$ via the recursion

$$
\begin{aligned}
& \left(d_{t} u^{k}, v\right)_{*}+\left(\left[u^{k}\right]^{\prime \prime}, v^{\prime \prime}\right) \\
& \quad=\gamma\left(\left[u^{k-1}\right]^{\prime \prime} \cdot n_{S}\left(u^{k-1}\right), v^{\prime \prime} \cdot n_{S}\left(u^{k-1}\right)+\left[u^{k-1}\right]^{\prime \prime} \cdot n_{S}^{\prime}\left(u^{k-1}\right) v\right)
\end{aligned}
$$

subject to $d_{t} u^{k}, v \in \mathcal{F}\left[u^{k-1}\right]$. Under moderate conditions on the step size $\tau$ in terms of $\gamma$ we obtain a monotonicity property for the iteration.

1.3. Conical sheets. Motivated by the problem of understanding folding and crumpling deformations of thin elastic sheets, the articles CM05, BKN13, MO14, Olb16; FM18 address the situation in which an elastic plate is placed on a circular obstacle of radius $r$ and then indented by an amount $\delta$ at the center $C$. The resulting deformation is homogeneous along rays starting from the center, points at a distance $\left(r^{2}+\delta^{2}\right)^{1 / 2}$ from the center are either in contact with the obstacle or above it. The displacement of these points entirely determines the full deformation of the sheet and it therefore suffices to compute the deformation of the points belonging to this circle. The displaced points belong to a sphere and are constrained by the obstacle. By an appropriate rescaling we may assume that $r^{2}+\delta^{2}=1$. A cross section of the rotationally symmetric setting through the center $C$ is depicted in Figure 1. The solutions of the two-dimensional problem and its one-dimensional reduction cannot be rotationally symmetric unless the indendation depth $\delta$ is trivial. 
The corresponding reduced description has been rigorously identified in FM18 and characterizes the deformation $u: S^{1} \rightarrow \mathbb{R}^{3}$ of the unit circle $S^{1} \subset \mathbb{R}^{2}$ via a minimization of the functional

$$
I[u]=\frac{1}{2} \int_{S^{1}} \kappa_{g}^{2} \mathrm{~d} x
$$

in the set of periodic curves $u \in H^{2}\left(S^{1} ; \mathbb{R}^{3}\right)$ subject to the constraints that $u$ attains its values on the unit sphere $S=S^{2} \subset \mathbb{R}^{3}$ and is inextensible, i.e.,

$$
|u(x)|^{2}=1, \quad\left|u^{\prime}(x)\right|^{2}=1,
$$

and that the curve does not penetrate the obstacle, i.e., for the vertical component $u_{3}$ of $u$ we have

$$
u_{3}(x) \geq \delta,
$$

for all $x \in S^{1}$. Because of the unit-length constraints on $u$ and $u^{\prime}$ we have that the normal curvature $\kappa_{n}$ of $u$ is given by

$$
\kappa_{n}=u^{\prime \prime} \cdot u=\left(u^{\prime} \cdot u\right)^{\prime}-\left|u^{\prime}\right|^{2}=-1,
$$

so that for the geodesic part we have

$$
\kappa_{g}^{2}=\kappa^{2}-\kappa_{n}^{2}=\left|u^{\prime \prime}\right|^{2}-1
$$

The reduced indentation problem thus leads to the following minimization problem for a given indentation depth $\delta \geq 0$.

$\left(\mathrm{P}_{\text {ind }}\right)\left\{\begin{array}{l}\text { Find a minimizing curve } u \in H^{2}\left(S^{1} ; \mathbb{R}^{3}\right) \text { for } \\ I[u]=\frac{1}{2} \int_{S^{1}}\left|u^{\prime \prime}\right|^{2} \mathrm{~d} s-\pi \\ \text { subject to }|u(x)|^{2}=1,\left|u^{\prime}(x)\right|^{2}=1, u_{3}(x) \geq \delta \text { for all } x \in S^{1} .\end{array}\right.$

Various features of minimizers have been characterized in [FM18], e.g., that the non-contact zone $\left\{s \in S^{1}: u_{3}(s)>\delta\right\}$ is an interval. Via less rigorous arguments it has been stated in [CM05] that minimizers have, in a certain projection, a unique maximum, i.e., that single folds of the indented sheet are preferred over double folds, as is observed in reality. To investigate such questions via numerical experiments we approximate the problem by imposing the inequality constraint using a penalty approximation, i.e., we consider

$$
I_{\varepsilon}[u]=\frac{1}{2} \int_{S^{1}}\left|u^{\prime \prime}\right|^{2} \mathrm{~d} x+\frac{1}{2 \varepsilon} \int_{S^{1}}\left(u_{3}-\delta\right)_{-}^{2} \mathrm{~d} x-\pi .
$$

The minimimization of $I_{\varepsilon}$ is done with a gradient flow that linearizes the constraints and which uses an implicit-explicit treatment of the penalty term defined via the convex-concave splitting

$$
(s-\delta)_{-}^{2}=(s-\delta)^{2}-(s-\delta)_{+}^{2}
$$

i.e., we compute a sequence $\left(u^{k}\right)_{k=0,1, \ldots}$ via

$$
\left(d_{t} u^{k}, v\right)_{*}+\left(\left[u^{k}\right]^{\prime \prime}, v^{\prime \prime}\right)+\varepsilon^{-1}\left(u_{3}^{k}-\delta, v_{3}\right)=\varepsilon^{-1}\left(\left(u_{3}^{k-1}-\delta\right)_{+}, v_{3}\right)
$$


for all $v \in H^{2}\left(S^{1} ; \mathbb{R}^{3}\right)$ subject to the linearized unit-length constraints and periodicity conditions contained in the space $\mathcal{F}\left[u^{k-1}\right]$

$$
d_{t} u^{k}, v \in \mathcal{F}\left[u^{k-1}\right] .
$$

The resulting iterative method is unconditionally energy monotone and converges to stationary configurations of low bending energy.

1.4. Outline. The article is organized as follows. In Section 2 we introduce the finite element spaces used to approximate $H^{2}$ curves and prove $\Gamma$-convergence results for the model problems. Section 3 is devoted to the development of stable gradient flow discretizations used to compute stationary configurations. In Section 4 we illustrate the theoretical findings by numerical experiments.

\section{Discretization And $\Gamma$-COnvergence}

In this section we define suitable finite element spaces to approximate curves, devise discretizations of the constrained minimization problems, and prove their variational convergence as discretization parameters tend to zero.

2.1. Finite element spaces. We discretize the constrained minimization problems using $H^{2}$ conforming finite element spaces for partitions

$$
0=z_{0}<z_{1}<\cdots<z_{J}=L
$$

of the interval $(0, L)$ with maximal mesh size $h=\max _{j=1, \ldots, J}\left|z_{j}-z_{j-1}\right|$ of the subintervals $I_{j}=\left[z_{j-1}, z_{j}\right]$. A finite element space subordinated to this partitioning is defined by imposing continuity and differentiability of the piecewise cubic curves at the nodes, i.e., we set

$$
V_{h}=\left\{v_{h} \in C^{1}\left(0, L ; \mathbb{R}^{3}\right):\left.v_{h}\right|_{I_{j}} \in \mathcal{P}_{3}\left(I_{j}\right)^{3}, j=1,2, \ldots, J\right\},
$$

where $\mathcal{P}_{\ell}(I)$ denotes the set of polynomials of maximal degree $\ell \geq 0$ on an interval $I$. The degrees of freedom in the space $V_{h}$ are the function values and derivatives at the nodes, i.e.,

$$
\left(v_{h}\left(z_{j}\right), v_{h}^{\prime}\left(z_{j}\right)\right)_{j=0, \ldots, J}
$$

Correspondingly, an interpolation operator $\mathcal{I}_{h}^{3,1}: H^{2}\left(0, L ; \mathbb{R}^{3}\right) \rightarrow V_{h}$ is defined by requiring that

$$
\mathcal{I}_{h}^{3,1} v\left(z_{j}\right)=v\left(z_{j}\right), \quad\left[\mathcal{I}_{h}^{3,1} v\right]^{\prime}\left(z_{j}\right)=v^{\prime}\left(z_{j}\right)
$$

for $j=0,1, \ldots, J$. We note that we have the interpolation estimates

$$
\left\|\left(\mathcal{I}_{h}^{3,1} v-v\right)^{(k)}\right\|_{L^{p}(0, L)} \leq c h^{3-k}\|v\|_{W^{3, p}(0, L)}
$$

for all $v \in W^{3, p}(0, L)$ and $k \leq 2$, cf. BS08. We also employ the standard piecewise linear interpolation operator

$$
\mathcal{I}_{h}: C^{0}([0, L]) \rightarrow W_{h}
$$

which is defined by requiring

$$
\mathcal{I}_{h} w\left(z_{j}\right)=w\left(z_{j}\right)
$$


for $j=0,1, \ldots, J$ and thereby defines an element in the space

$$
W_{h}=\left\{w_{h} \in C^{0}([0, L]):\left.w_{h}\right|_{I_{j}} \in \mathcal{P}_{1}\left(I_{j}\right), j=1,2, \ldots, J\right\} .
$$

For the interpolation operator we have that

$$
\left\|\mathcal{I}_{h} w-w\right\|_{L^{p}(0, L)} \leq c h\left\|w^{\prime}\right\|_{L^{p}(0, L)},
$$

if $p>1$. With the interpolation operator $\mathcal{I}_{h}$ we define discrete inner products and norms via

$$
(v, w)_{h}=\int_{0}^{L} \mathcal{I}_{h}[v w] \mathrm{d} x, \quad\|v\|_{L_{h}^{p}(0, L)}^{p}=\int_{0}^{L} \mathcal{I}_{h}\left[|v|^{p}\right] \mathrm{d} x
$$

for $v, w \in C\left([0, L] ; \mathbb{R}^{\ell}\right)$ and $1 \leq p \leq \infty$, where $\|v\|_{L_{h}^{\infty}(0, L)}=\max _{j=0, \ldots, J}\left|v\left(z_{h}\right)\right|$.

2.2. Discrete minimization problems. The pointwise constraints and the nonlinearities require making certain approxiomations which lead to inconsistency terms. We impose the arclength condition and the surface constraints at the nodes of a partitioning, i.e., we impose that

$$
\mathcal{I}_{h}\left|v_{h}^{\prime}\right|^{2}=1, \quad \mathcal{I}_{h} \Phi_{S}\left(v_{h}\right)=0,
$$

which is equivalent to the nodal constraints

$$
\left|v_{h}^{\prime}\left(z_{j}\right)\right|=1, \quad v_{h}\left(z_{j}\right) \in S
$$

for $j=0,1, \ldots, J$. The discrete set of admissible curves is then given by

$$
\mathcal{A}_{h}=\left\{v_{h} \in V_{h}: \mathcal{I}_{h}\left|v_{h}^{\prime}\right|^{2}=1, \mathcal{I}_{h} \Phi_{S}\left(v_{h}\right)=0, L_{\mathrm{bc}}\left[v_{h}\right]=\ell_{\mathrm{bc}}\right\} .
$$

It provides an approximation of the continuous set of admissible curves defined as

$$
\mathcal{A}=\left\{v \in H^{2}\left(0, L ; \mathbb{R}^{3}\right):\left|v^{\prime}\right|^{2}=1, \Phi_{S}(v)=0, L_{\mathrm{bc}}[v]=\ell_{\mathrm{bc}}\right\} .
$$

We note that if the continuous admissible set is nonempty then also the discrete admissible set is nonempty, i.e., we have the implication

$$
v \in \mathcal{A} \Longrightarrow \mathcal{I}_{h}^{3,1} v \in \mathcal{A}_{h},
$$

where we assume that $L_{\mathrm{bc}}[v]$ only depends on the boundary values of $v$ and $v^{\prime}$. Our convergence result considers the minimization of

$$
I_{\gamma}[u]= \begin{cases}\frac{1}{2} \int_{0}^{L}\left|u^{\prime \prime}\right|^{2}-\gamma\left|u^{\prime \prime} \cdot n_{S}(u)\right|^{2} \mathrm{~d} x & \text { for } u \in \mathcal{A}, \\ +\infty & \text { for } H^{2}\left(0, L ; \mathbb{R}^{3}\right) \backslash \mathcal{A},\end{cases}
$$

with a parameters $\gamma \in[0,1)$. The approximating discrete functionals are given by

$$
I_{\gamma, h}\left[u_{h}\right]= \begin{cases}\frac{1}{2} \int_{0}^{L}\left|u_{h}^{\prime \prime}\right|^{2}-\gamma\left|u_{h}^{\prime \prime} \cdot n_{S}\left(u_{h}\right)\right|^{2} \mathrm{~d} x & \text { for } u_{h} \in \mathcal{A}_{h}, \\ +\infty & \text { for } H^{2}\left(0, L ; \mathbb{R}^{3}\right) \backslash \mathcal{A}_{h},\end{cases}
$$

for $u_{h} \in \mathcal{A}_{h}$ with the extension by $+\infty$ on $H^{2}\left(0, L ; \mathbb{R}^{3}\right) \backslash \mathcal{A}_{h}$. To prove the convergence $I_{\gamma, h} \rightarrow I_{\gamma}$ we impose a definiteness property on the boundary condition operator $L_{\mathrm{bc}}$ and an approximability condition on $\mathcal{A}$. 
Assumption 2.1 (Definiteness). The seminorm $v \mapsto\left\|v^{\prime \prime}\right\|$ is a norm on the kernel of the operator $L_{\mathrm{bc}}: H^{2}\left(0, L ; \mathbb{R}^{3}\right) \rightarrow \mathbb{R}^{\ell}$.

The assumption is satisfied for clamped boundary conditions, e.g., $L_{\mathrm{bc}}[v]=$ $\left(v(0), v^{\prime}(0)\right)$, and boundary conditions that fix both end points, i.e., $L_{\mathrm{bc}}[v]=$ $(v(0), v(L))$. We always assume that the boundary conditions lead to a nonempty set $\mathcal{A}$.

Assumption 2.2 (Density of smooth curves). The subset of smooth curves $\mathcal{A} \cap H^{3}\left(0, L ; \mathbb{R}^{3}\right)$ is dense in $\mathcal{A}$ with respect to strong convergence in $H^{2}$.

A relaxation of the assumption is discussed below in Remark 2.4. The assumption can be justified by regularizing curves in $\mathcal{A}$, projecting regular curves on $S$, adjusting the boundary conditions, and carrying out a suitable reparametrization. We refer the reader to BR19] for related ideas.

Proposition 2.3 ( $\Gamma$-convergence). If $0 \leq \gamma<1, \Phi_{S} \in C^{1}\left(\mathbb{R}^{3}\right)$ and Assumptions 2.1 and 2.2 are satisfied then we have $I_{\gamma, h} \rightarrow I_{\gamma}$ in the sense of $\Gamma$-convergence with respect to weak convergence in $\mathrm{H}^{2}$, i.e., we have the following:

(i) If $\left(u_{h}\right)_{h>0} \subset H^{2}\left(0, L ; \mathbb{R}^{3}\right)$ such that $u_{h} \in \mathcal{A}_{h}$ for every $h>0$ and $I_{\gamma, h}\left[u_{h}\right] \leq c$ then there exists $u \in \mathcal{A}$ such that $u_{h} \rightarrow u$ in $H^{2}$ and

$$
I_{\gamma}[u] \leq \liminf _{h \rightarrow 0} I_{\gamma, h}\left[u_{h}\right]
$$

(ii) For every $u \in \mathcal{A}$ there exists a sequence $\left(u_{h}\right)_{h>0} \subset H^{2}\left(0, L ; \mathbb{R}^{3}\right)$ such that $u_{h} \rightarrow u$ in $H^{2}$ and

$$
I_{\gamma}[u]=\lim _{h \rightarrow 0} I_{\gamma, h}\left[u_{h}\right]
$$

(iii) Weak accumulation points of sequences of quasiminimizers $\left(u_{h}\right)_{h>0}$ for the functionals $I_{\gamma, h}$ in $H^{2}$ are minimizers for $I_{\gamma}$.

Proof. (i) If $I_{\gamma, h}\left[u_{h}\right] \leq c$ for a sequence $\left(u_{h}\right)_{h>0}$ then, since $\gamma<1$ and since

$$
\begin{aligned}
& \left|u_{h}^{\prime \prime}\right|^{2}-\gamma\left|u_{h}^{\prime \prime} \cdot n_{S}\left(u_{h}\right)\right|^{2} \\
& \quad=(1-\gamma)\left|u_{h}^{\prime \prime}\right|^{2}+\gamma\left|\left(I_{3}-n_{S}\left(u_{h}\right) \otimes n_{S}\left(u_{h}\right)\right) u_{h}^{\prime \prime}\right|^{2},
\end{aligned}
$$

we have that the sequence is bounded in $H^{2}\left(0, L ; \mathbb{R}^{3}\right)$ and there exists a weak limit $u \in H^{2}\left(0, L ; \mathbb{R}^{3}\right)$ of an appropriate subsequence which is not relabeled. The boundedness of the linear operator $L_{\mathrm{bc}}[v]$ shows that we have $L_{\mathrm{bc}}[u]=\ell_{\mathrm{bc}}$. The compactness of the embedding $H^{2}(0, L) \rightarrow W^{1, \infty}(0, L)$ implies that the sequence $\left(u_{h}^{\prime}\right)_{h>0}$ is strongly convergent in $L^{\infty}\left(0, L ; \mathbb{R}^{3}\right)$. Using that $\mathcal{I}_{\gamma, h}\left|u_{h}^{\prime}\right|^{2}=1$ we thus deduce that

$$
\begin{aligned}
\left\|\left|u_{h}^{\prime}\right|^{2}-1\right\|_{L^{2}(0, L)} & =\left\|\left|u_{h}^{\prime}\right|^{2}-\mathcal{I}_{h}\left|u_{h}^{\prime}\right|^{2}\right\|_{L^{2}(0, L)} \\
& \leq 2 c h\left\|u_{h}^{\prime}\right\|_{L^{\infty}(0, L)}\left\|u_{h}^{\prime \prime}\right\|_{L^{2}(0, L)}^{2}
\end{aligned},
$$


which implies that $\left|u_{h}^{\prime}\right|^{2} \rightarrow 1$ in $L^{2}(0, L)$. We have that

$$
\begin{aligned}
\left\|\Phi_{S}\left(u_{h}\right)\right\|_{L^{\infty}(0, L)} & =\left\|\Phi_{S}\left(u_{h}\right)-\mathcal{I}_{h} \Phi_{S}\left(u_{h}\right)\right\|_{L^{\infty}(0, L)} \\
& \leq \operatorname{ch}\left\|\Phi_{S}^{\prime}\left(u_{h}\right) u_{h}^{\prime}\right\|_{L^{\infty}(0, L)} .
\end{aligned}
$$

The pointwise convergence $u_{h} \rightarrow u$ and continuity of $\Phi_{S}$ imply that $\Phi_{S}(u)=$ 0 in $(0, L)$. Hence, we have that $u \in \mathcal{A}$. Since

$$
P_{u_{h}}=I_{3}-n_{S}\left(u_{h}\right) \otimes n_{S}\left(u_{h}\right) \rightarrow P_{u}=I_{3}-n_{S}(u) \times n_{S}(u)
$$

strongly in $L^{\infty}\left(0, L ; \mathbb{R}^{3 \times 3}\right)$ it follows that $P_{u_{h}} u_{h}^{\prime \prime} \rightarrow P_{u} u^{\prime \prime}$ in $L^{2}\left(0, L ; \mathbb{R}^{3}\right)$ and the weak lower semicontinuity of the $L^{2}$ norm in combination with the identity (1) shows that

$$
\int_{0}^{L}\left|u^{\prime \prime}\right|^{2}-\gamma\left|u^{\prime \prime} \cdot n_{S}(u)\right|^{2} \mathrm{~d} x \leq \liminf _{h \rightarrow 0} \int_{0}^{L}\left|u_{h}^{\prime \prime}\right|^{2}-\gamma\left|u_{h}^{\prime \prime} \cdot n_{S}\left(u_{h}\right)\right|^{2} \mathrm{~d} x
$$

i.e., that $I_{\gamma}[u] \leq \liminf _{h \rightarrow 0} I_{\gamma, h}\left[u_{h}\right]$.

(ii) Since $I_{\gamma}$ is continuous on $\mathcal{A}$ with respect to strong convergence in $H^{2}$ and because of Assumption 2.2, we may assume that $u \in \mathcal{A} \cap H^{3}\left(0, L ; \mathbb{R}^{3}\right)$. Letting $u_{h}=\mathcal{I}_{h}^{3,1} u$ we have that $u_{h} \in \mathcal{A}_{h}, u_{h} \rightarrow u$ in $H^{2}$, and $I_{\gamma}[u]=$ $\lim _{h \rightarrow 0} I_{\gamma, h}\left[u_{h}\right]$.

(iii) The convergence of quasi-minimizers is an immediate consequence of the equicoercivity of the functionals $I_{\gamma, h}$ owing to the condition $\gamma<1$ and assertions (i) and (ii).

Remark 2.4. To avoid Assumption 2.2 one may impose the arclength and surface constraints in a relaxed sense in defining $\mathcal{A}_{h}$, i.e., using

$$
\begin{aligned}
\widetilde{\mathcal{A}}_{h}=\left\{v_{h} \in V_{h}:, L_{\mathrm{bc}}\left[v_{h}\right]=\ell_{\mathrm{bc}},\right. \\
\\
\left.\left\|\mathcal{I}_{h}\left|v_{h}^{\prime}\right|^{2}-1\right\|_{L^{\infty}(0, L)} \leq \alpha_{h},\left\|\mathcal{I}_{h} \Phi_{S}\left(v_{h}\right)\right\|_{L^{\infty}(0, L)} \leq \beta_{h}\right\},
\end{aligned}
$$

with $h$-dependent parameters $\alpha_{h}, \beta_{h}>0$. In this case, one may construct a recovery sequence $u_{h}$ in part (ii) of the Proposition by letting $\widetilde{u} \in C^{\infty}\left(0, L ; \mathbb{R}^{3}\right)$ be a regularization of $u \in \mathcal{A}$ which obeys the boundary conditions and define $u_{h}=\mathcal{I}_{h}^{3,1} u$. If $\alpha_{h}, \beta_{h}$ are appropriately chosen we have $u_{h} \in \widetilde{\mathcal{A}}_{h}$ and $u_{h} \rightarrow u$ in $H^{2}$.

2.3. Application to model problems. We next apply the abstract $\Gamma$ convergence result to the model problems defined by the variational problems $\left(\mathrm{P}_{\text {bend }}\right),\left(\overline{\mathrm{P}_{\text {geod }}^{\gamma}}\right)$, and $\left(\overline{\mathrm{P}_{\text {ind }}}\right)$. We assume throughout the following that $\Phi_{S} \in C^{1}\left(\mathbb{R}^{3}\right)$ and that Assumptions 2.1 and 2.2 are satisfied and always consider weak convergence in $H^{2}$. The discretization of the constrained nonlinear bending problem $\left(\mathrm{P}_{\text {bend }}\right)$ is defined as:

$\left(\mathrm{P}_{\text {bend }}^{h}\right) \quad\left\{\begin{array}{l}\text { Find a minimizing curve } u_{h} \in \mathcal{A}_{h} \text { for } \\ I\left[u_{h}\right]=\frac{1}{2} \int_{0}^{L}\left|u_{h}^{\prime \prime}\right|^{2} \mathrm{~d} x .\end{array}\right.$

A convergence result is obtained from choosing $\gamma=0$ in Proposition 2.3 . 
Corollary 2.5 (Constrained nonlinear bending). The minimization problems ( $\left.\mathrm{P}_{\text {bend }}^{h}\right)$ approximate the problem $\left(\mathrm{P}_{\text {bend }}\right)$ as $h \rightarrow 0$.

A discretization of the geodesic curvature minimization problem $\left(\mathrm{P}_{\text {geod }}^{\gamma}\right.$ is defined as:

$\left(\mathrm{P}_{\text {geod }}^{\gamma, h}\right)$

$$
\left\{\begin{array}{l}
\text { Find a minimizing curve } u_{h} \in \mathcal{A}_{h} \text { for } \\
I_{\gamma, h}\left[u_{h}\right]=\frac{1}{2} \int_{0}^{L}\left|u_{h}^{\prime \prime}\right|^{2}-\gamma\left|u_{h}^{\prime \prime} \cdot n_{S}\left(u_{h}\right)\right|^{2} \mathrm{~d} x .
\end{array}\right.
$$

This problem approximates for fixed $0<\gamma<1$ the stabilized problem $\left(\mathrm{P}_{\text {geod }}^{\gamma}\right.$ which is a direct consequence of Proposition 2.3. We also have that the regularized minimization problems converge for $\gamma \rightarrow 1$ to the original, unstabilized problem defined with $\gamma=1$.

Corollary 2.6 (Geodesic curvature minimization). The minimization problems $\sqrt{\mathrm{P}_{\text {geod }}^{\gamma, h}}$ approximate problem $\left(\overline{\mathrm{P}_{\text {geod }}^{\gamma}}\right)$ as $h \rightarrow 0$. For $\gamma \rightarrow 1$ problems $\left(\overline{\mathrm{P}_{\text {geod }}^{\gamma}}\right)$ approximate problem $\left(\overline{\mathrm{P}_{\text {geod }}^{\gamma}}\right)$ with $\gamma=1$.

Proof. The first part follows from Proposition 2.3. To prove the second part one uses that second derivatives of arclength-parametrized curves on $S$ are bounded by their geodesic curvature.

Remarks 2.7. (i) For an efficient numerical realization it is helpful to replace the function $u_{h}^{\prime \prime} \cdot n_{S}\left(u_{h}\right)$ by $u_{h}^{\prime \prime} \cdot n_{S}\left(\bar{u}_{h}\right)$, where $\bar{u}_{h}$ is a piecewise constant approximation of $u_{h}$. The approximation result remains valid if $u_{h}-\bar{u}_{h} \rightarrow 0$ in $L^{\infty}\left(0, L ; \mathbb{R}^{3}\right)$ for every bounded seqence $\left(u_{h}\right)_{h>0}$ in $H^{2}\left(0, L ; \mathbb{R}^{3}\right)$, e.g., if $\bar{u}_{h}$ is defined via the midpoint values of $u_{h}$.

(ii) A modification of the method is necessary to justify a joint limit passage $(h, \gamma) \rightarrow(0,1)$. In particular, control on the normal part of $u_{h}^{\prime \prime}$ is needed, e.g., via requiring that $u_{h}^{\prime}\left(z_{j}\right)$ is a tangent vector at every node $z_{j}$, $j=0,1, \ldots, J$.

A discretization of the sheet indentation problem $\left(\overline{\mathrm{P}_{\text {ind }}}\right)$ is defined as:

$\left(\mathrm{P}_{\text {ind }}^{h, \varepsilon}\right)$

$$
\left\{\begin{array}{l}
\text { Find a minimizing curve } u_{h} \in \mathcal{A}_{h} \text { for } \\
I_{h, \varepsilon}\left[u_{h}\right]=\frac{1}{2} \int_{0}^{L}\left|u_{h}^{\prime \prime}\right|^{2}+\frac{1}{2 \varepsilon} \int_{0}^{L} \mathcal{I}_{h}\left(u_{3, h}-\delta\right)_{-}^{2} \mathrm{~d} x .
\end{array}\right.
$$

A convergence result is obtained from choosing $\gamma=0$ in Proposition 2.3 and showing that the penalty term turns into a rigid constraint as $(h, \varepsilon) \rightarrow 0$.

Corollary 2.8 (Constrained nonlinear bending). Assume that Assumption 2.2 holds with $\mathcal{A}$ replaced by the set of functions $u \in \mathcal{A}$ with $u_{3} \geq \delta$. Then the minimization problems $\left(\mathrm{P}_{\mathrm{ind}}^{h, \varepsilon}\right)$ approximate the problem $\left(\mathrm{P}_{\mathrm{ind}}\right)$ as $(h, \varepsilon) \rightarrow 0$.

Proof. Certain modifications of the proof of Proposition 2.3 are required. If the sequence $\left(u_{h}\right)_{h>0}$ is such that $I_{h, \varepsilon}\left[u_{h}\right] \leq c$ then we have $\|\left(u_{3, h}-\right.$ $\delta)_{-} \|_{L_{h}^{2}(0, L)}^{2} \leq 2 c \varepsilon$ and every weak accumulation point $u \in H^{2}\left(0, L ; \mathbb{R}^{3}\right)$ 
satisfies $u_{3} \geq \delta$. Since the penalty term is nonnegative we have that $\liminf _{(h, \varepsilon) \rightarrow 0} I_{h, \varepsilon}\left[u_{h}\right] \geq I[u]$. For a curve $u \in \mathcal{A} \cap C^{\infty}\left(0, L ; \mathbb{R}^{3}\right)$ obeying the constraint $u_{3} \geq \delta$ we have that the interpolants $u_{h}=\mathcal{I}_{h}^{3,1} u$ also satisfy $\mathcal{I}_{h} u_{3, h} \geq \delta$ so that the penalty term in the functional disappears and the second part of the proof of Proposition 2.3 applies verbatimly.

\section{Discrete GRADient Flows on SURFACES}

We investigate in this section the stability of gradient flows for curvature energies defined on classes of arclength parametrized curves that belong to a given surface. The first model uses the full bending energy, the second one is defined by the geodesic curvature, while the third problem involves an obstacle constraint.

3.1. Constrained elastic flow of curves. Minimizing the bending energy of curves restricted to a surface $S$ subject to inextensibility and boundary conditions as formulated in problem ( $\mathrm{P}_{\text {bend }}$ leads to gradient flows such as

$$
\partial_{t} u=-u^{(4)}+\left(\lambda u^{\prime}\right)^{\prime}+\mu \Phi_{S}^{\prime}(u),
$$

where $\lambda$ and $\mu$ are Lagrange multipliers related to inextensibility and surface constraints. More generally, given a metric $(\cdot, \cdot)_{*}$ defined on $L^{2}\left(0, L ; \mathbb{R}^{3}\right)$ we consider the evolution problem

$$
\left(\partial_{t} u, v\right)_{*}+\left(u^{\prime \prime}, v^{\prime \prime}\right)=0
$$

that determines a family $u:[0, T] \rightarrow H^{2}\left(0, L ; \mathbb{R}^{3}\right)$ of curves satisfying

$$
u(0)=u_{0}, \quad u(t) \in \mathcal{A}
$$

for all $t \in[0, T]$. We require the test functions $v \in H^{2}\left(0, L ; \mathbb{R}^{3}\right)$ to belong to the linearization of $\mathcal{A}$ at $u(t)$, i.e., that $v \in \mathcal{F}[u(t)]$, where

$$
\mathcal{F}[\widehat{u}]=\left\{v \in H^{2}\left(0, L ; \mathbb{R}^{3}\right): \Phi_{S}^{\prime}(\widehat{u}) \cdot v=0, \quad \widehat{u}^{\prime} \cdot v=0, \quad L_{\mathrm{bc}}[v]=0\right\} .
$$

Note that also $\partial_{t} u(t) \in \mathcal{F}[u(t)]$. To discretize the evolution equation we use a step size $\tau>0$ and the backward difference operator

$$
d_{t} u^{k}=\frac{1}{\tau}\left(u^{k}-u^{k-1}\right)
$$

For a partition $z_{0}<z_{1}<\cdots<z_{J}$ of $(0, L)$ we define the discrete linearized admissible space

$$
\mathcal{F}_{h}\left[\widehat{u}_{h}\right]=\left\{v_{h} \in V_{h}: \mathcal{I}_{h}\left[\Phi_{S}^{\prime}\left(\widehat{u}_{h}\right) \cdot v_{h}\right]=0, \mathcal{I}_{h}\left[\widehat{u}_{h}^{\prime} \cdot v_{h}^{\prime}\right]=0, L_{\mathrm{bc}}\left[v_{h}\right]=0\right\},
$$

i.e., the orthogonality relations are imposed only at the nodes $z_{0}, z_{z}, \ldots, z_{J}$, in accordance with the definition of the discrete admissible set $\mathcal{A}_{h}$. This leads to the following algorithm.

Algorithm 3.1 (Constrained curvature flow). Choose $u_{h}^{0} \in V_{h}$ such that $\mathcal{I}_{h} \Phi_{S}\left(u_{h}^{0}\right)=0$ and $\mathcal{I}_{h}\left|\left[u^{0}\right]^{\prime}\right|^{2}=1$ and $L_{\mathrm{bc}}\left[u_{h}^{0}\right]=\ell_{\mathrm{bc}}$. Set $k=0$.

(1) Compute $d_{t} u_{h}^{k} \in V_{h}$ such that

$$
\left(d_{t} u_{h}^{k}, v_{h}\right)_{*}+\left(\left[u_{h}^{k-1}+\tau d_{t} u_{h}^{k}\right]^{\prime \prime}, v_{h}^{\prime \prime}\right)=0
$$


for all $v_{h} \in V_{h}$ subject to the constraints

$$
d_{t} u_{h}^{k}, v_{h} \in \mathcal{F}_{h}\left[u_{h}^{k-1}\right] .
$$

(2) Define $u_{h}^{k}=u_{h}^{k-1}+\tau d_{t} u_{h}^{k}$; set $k \rightarrow k+1$, and continue with (1).

The iteration of Algorithm 3.1 is unconditionally well defined and energy decreasing and leads to a violation of the constraints that is controlled by the step size $\tau>0$.

Proposition 3.2. (i) Algorithm 3.1 defines a sequence $\left(u_{h}^{k}\right)_{k=0,1, \ldots} \subset V_{h}$ such that for every $K \geq 0$ we have

$$
I\left[u_{h}^{K}\right]+\tau \sum_{k=1}^{K}\left\|d_{t} u_{h}^{k}\right\|_{*}^{2} \leq I\left[u_{h}^{0}\right]=e_{0, h} .
$$

(ii) Assume that $u_{h}^{0} \in \mathcal{A}_{h}$ and that the inner product $(\cdot, \cdot)_{*}$ induces a norm $\|\cdot\|_{*}$ with

$$
\left\|v_{h}^{\prime}\right\|_{L_{h}^{\infty}(0, L)}^{2}=\left\|\mathcal{I}_{h} v_{h}^{\prime}\right\|_{L^{\infty}(0, L)}^{2} \leq c_{*}\left\|v_{h}\right\|_{*}^{2}
$$

for all $v_{h} \in V_{h}$ and $\left|\Phi_{S}^{\prime \prime}(s)\right| \leq c_{S, 2}\left(1+|s|^{r}\right)$ for all $s \in \mathbb{R}^{3}$. Then, we have for every $K \geq 0$ that

$$
\max _{k=0,1, \ldots, K}\left\|\left|\left[u_{h}^{k}\right]^{\prime}\right|^{2}-1\right\|_{L_{h}^{\infty}(0, L)} \leq c_{*} \tau e_{0, h},
$$

and

$$
\max _{k=0,1, \ldots, K}\left\|\Phi_{S}\left(u_{h}^{k}\right)\right\|_{L_{h}^{\infty}(0, L)} \leq c_{*} c_{S, 2} c^{\prime} \tau e_{0, h}^{r+1} .
$$

Proof. We test the formulation of Step (1) of Algorithm 3.1 with $v_{h}=d_{t} u_{h}^{k}$ to deduce with a binomial formula that

$$
\left\|d_{t} u_{h}^{k}\right\|_{*}^{2}+d_{t} \frac{1}{2}\left\|\left[u_{h}^{k}\right]^{\prime \prime}\right\|^{2}+\frac{\tau}{2}\left\|\left[d_{t} u_{h}^{k}\right]^{\prime \prime}\right\|^{2}=0 .
$$

A summation over $k=1,2, \ldots, K$ yields the asserted energy estimate. The nodewise orthogonality $\left[d_{t} u_{h}^{k}\right]^{\prime} \cdot\left[u_{h}^{k-1}\right]^{\prime}=0$ and the relation $u_{h}^{k}=u_{h}^{k-1}+\tau d_{t} u_{h}^{k}$ imply that at every node $z \in \mathcal{N}_{h}$ we have

$$
\left|\left[u_{h}^{k}\right]^{\prime}\right|^{2}=\left|\left[u_{h}^{k-1}\right]^{\prime}\right|^{2}+\tau^{2}\left|\left[d_{t} u_{h}^{k}\right]^{\prime}\right|^{2}=\cdots=1+\tau^{2} \sum_{\ell=1}^{k}\left|\left[d_{t} u_{h}^{k}\right]^{\prime}\right|^{2} .
$$

The energy bound and the assumed inequality for $\|\cdot\|_{*}$ imply the bound for the arclength violation. For the surface constraint we note that the application of a Taylor formula and the fact that $d_{t} u_{h}^{k} \in \mathcal{F}_{h}\left[u_{h}^{k-1}\right]$ yield that at every node we have

$$
\Phi_{S}\left(u_{h}^{k}\right)=\Phi_{S}\left(u_{h}^{k-1}\right)+\frac{1}{2} \tau^{2} \Phi_{S}^{\prime \prime}\left(\xi_{h}^{k}\right)\left[d_{t} u_{h}^{k}, d_{t} u_{h}^{k}\right]
$$


Repeating this argument and using $\Phi_{S}\left(u_{h}^{0}\right)=0$ at the nodes we infer with the assumed estimate for $\Phi_{S}^{\prime \prime}$ that

$$
\left\|\mathcal{I}_{h} \Phi_{S}\left(u_{h}^{k}\right)\right\|_{L^{\infty}(0, L)} \leq \frac{1}{2} \tau c_{S, 2}\left(1+\left\|\mathcal{I}_{h} \xi_{h}^{k}\right\|_{L^{\infty}(0, L)}^{r}\right) \tau \sum_{\ell=1}^{k}\left\|\mathcal{I}_{h} d_{t} u_{h}^{\ell}\right\|_{L^{\infty}(0, L)}^{2} .
$$

Since the nodal values of $\xi_{h}^{k}$ belongs to the line segment connecting $u_{h}^{k}$ and $u_{h}^{k-1}$ we may incorporate the discrete $L^{\infty}$ estimates to deduce the estimate for the nodewise surface constraint violation.

3.2. Geodesic curvature flow. To develop an iterative scheme for the approximate solution of the geodesic curvature problem $\left(\sqrt{\mathrm{P}_{\text {geod }}^{\gamma}}\right)$ we follow the ideas used for the constrained bending problem and use that

$$
\kappa_{g}^{2}=\left|u^{\prime \prime}\right|^{2}-\left|u^{\prime \prime} \cdot n_{S}(u)\right|^{2} .
$$

To control the nonlinear second term by the first one, we introduce a stabilization via a damping factor $\gamma_{\varepsilon}=\left(1-\varepsilon^{2}\right)$. This leads to the functional

$$
I_{\varepsilon}[u]=\frac{1}{2} \int_{0}^{L}\left|u^{\prime \prime}\right|^{2}-\gamma_{\varepsilon}\left|u^{\prime \prime} \cdot n_{S}(u)\right|^{2} \mathrm{~d} x .
$$

Because of the stabilization we have the implication

$$
I_{\varepsilon}[u] \leq c_{0} \quad \Longrightarrow \quad\left\|u^{\prime \prime}\right\|^{2} \leq 2 c_{0} \varepsilon^{-2} .
$$

While on the continuous level the geodesic curvature of a curve on the surface $S$ controls the full curvature this is not the case for the discretization and hence necessitates the stabilization. We assume that

$$
n_{S}: \mathbb{R}^{3} \rightarrow \mathbb{R}^{3}
$$

is a $C^{2}$ vector field which coincides with the normal field on $S$, i.e., we have $\left.n_{S}\right|_{S}=\frac{\Phi_{S}^{\prime}(u)}{\left|\Phi_{S}^{\prime}(u)\right|}$. We further assume that $n_{S}$ has bounded derivatives. To simplify notation we use the mapping

$$
G_{\varepsilon}[u]=\frac{\gamma_{\varepsilon}}{2} \int_{0}^{L}\left|u^{\prime \prime} \cdot n_{S}(u)\right|^{2} \mathrm{~d} x .
$$

The constrained gradient flow for $I_{\varepsilon}$ can thus be represented as

$$
\left(\partial_{t} u, v\right)_{*}+\left(u^{\prime \prime}, v^{\prime \prime}\right)=G_{\varepsilon}^{\prime}[u ; v]
$$

where

$$
G_{\varepsilon}^{\prime}[u ; v]=\gamma_{\varepsilon} \int_{0}^{L} u^{\prime \prime} \cdot n_{S}(u)\left(v^{\prime \prime} \cdot n_{S}(u)+u^{\prime \prime} \cdot n_{S}^{\prime}(u) v\right) \mathrm{d} x .
$$

We note that we have

$$
G_{\varepsilon}^{\prime}[u ; v] \leq \gamma_{\varepsilon}\left(\left\|u^{\prime \prime}\right\|\left\|v^{\prime \prime}\right\|+c_{n_{S}}\left\|u^{\prime \prime}\right\|^{2}\|v\|_{L^{\infty}(0, L)}\right) .
$$

For ease of presentation we consider a semi-discrete setting. All arguments carry over to the case of a spatially discrete scheme. 
Algorithm 3.3 (Constrained geodesic curvature flow). Choose $u^{0} \in V$ such that $\Phi_{S}\left(u^{0}\right)=0$ and $\left|\left[u^{0}\right]^{\prime}\right|^{2}=1$ and $L_{\mathrm{bc}}\left[u^{0}\right]=\ell_{\mathrm{bc}}$. Set $k=0$.

(1) Compute $d_{t} u^{k} \in V$ such that

$$
\left(d_{t} u^{k}, v\right)_{*}+\left(\left[u^{k-1}+\tau d_{t} u^{k}\right]^{\prime \prime}, v^{\prime \prime}\right)=G_{\varepsilon}^{\prime}\left[u^{k-1} ; v\right]
$$

for all $v \in V$ subject to the constraints

$$
d_{t} u^{k}, v \in \mathcal{F}\left[u^{k-1}\right] .
$$

(2) Define $u^{k}=u^{k-1}+\tau d_{t} u^{k}$; set $k \rightarrow k+1$, and continue with (1).

We have the following stability properties for Algorihm 3.3 .

Proposition 3.4. Assume that there exists $c_{*}>0$ such that

$$
\|v\|_{L^{\infty}(0, L)}+\left\|v^{\prime \prime}\right\| \leq c_{*}\|v\|_{*}
$$

for all $v \in V$.

(i) There exists $c_{3} \geq 0$ such that if $c_{3} \tau \varepsilon^{-1} \leq 1 / 2$ then the iterates of Algorithm 3.3 satisfy for all $K \geq 0$

$$
I_{\varepsilon}\left[u^{K}\right]+\left(1-c_{3} \tau \varepsilon^{-1}\right) \tau \sum_{k=1}^{K}\left\|d_{t} u^{k}\right\|_{*}^{2} \leq I_{\varepsilon}\left[u^{0}\right] .
$$

(ii) Under the above condition the bounds on the constraint violation errors apply as in Proposition 3.2 (ii).

Proof. We argue by induction and assume that the energy estimate and the constraint violation bounds have been established up to some number $k-1 \geq 0$ so that

$$
I_{\varepsilon}\left[u^{k-1}\right]+\frac{\tau}{2} \sum_{\ell=1}^{k-1}\left\|d_{t} u^{\ell}\right\|_{*}^{2} \leq I_{\varepsilon}\left[u^{0}\right]=e_{0} .
$$

This implies that

$$
\left\|\left[u^{k-1}\right]^{\prime \prime}\right\| \leq \sqrt{2} e_{0}^{1 / 2} \varepsilon^{-1} .
$$

By the assumption on the boundary data we thus have that $\left\|u^{k-1}\right\|_{H^{2}(0, L)} \leq$ $c_{1} \varepsilon^{-1}$. Moroever, we have that $\left\|u^{k-1}\right\|_{L^{\infty}(0, L)} \leq c$. To derive an auxiliary bound we choose $v=d_{t} u^{k}$ in Step (1) of Algorithm 3.3. Incorporating the bound for $G_{\varepsilon}^{\prime}$ and noting $\gamma_{\varepsilon} \leq 1$ this leads to

$$
\begin{aligned}
\left\|d_{t} u^{k}\right\|_{*}^{2} & +d_{t} \frac{1}{2}\left\|\left[u^{k}\right]^{\prime \prime}\right\|^{2}+\frac{\tau}{2}\left\|\left[d_{t} u^{k}\right]^{\prime \prime}\right\|^{2} \\
& \leq\left\|\left[u^{k-1}\right]^{\prime \prime}\right\|\left\|\left[d_{t} u^{k}\right]^{\prime \prime}\right\|+\left\|\left[u^{k-1}\right]^{\prime \prime}\right\|^{2} c_{n_{S}}\left\|d_{t} u^{k}\right\|_{L^{\infty}(0, L)}
\end{aligned}
$$

By the assumption on the inner product $(\cdot, \cdot)_{*}$ we have that

$$
\left\|d_{t} u^{k}\right\|_{L^{\infty}(0, L)}+\left\|\left[d_{t} u^{k}\right]^{\prime \prime}\right\| \leq c_{*}\left\|d_{t} u^{k}\right\|_{*}
$$

and we deduce that

$$
\frac{1}{2}\left\|d_{t} u^{k}\right\|_{*}^{2}+d_{t} \frac{1}{2}\left\|\left[u^{k}\right]^{\prime \prime}\right\|^{2} \leq c_{1} \varepsilon^{-2}
$$


Hence, by choosing $\tau$ sufficiently small, we have that

$$
\left\|\left[u^{k}\right]^{\prime \prime}\right\|^{2} \leq\left\|\left[u^{k-1}\right]^{\prime \prime}\right\|^{2}+2 \tau c_{1} \varepsilon^{-2} \leq 5 e_{0} \varepsilon^{-2} .
$$

We next improve the latter bound by choosing again $v=d_{t} u^{k}$ and using

$$
G_{\varepsilon}\left[u^{k}\right]-G_{\varepsilon}\left[u^{k-1}\right]=\tau G_{\varepsilon}^{\prime}\left[u^{k-1} ; d_{t} u^{k}\right]+\tau^{2} G_{\varepsilon}^{\prime \prime}\left[\xi^{k} ; d_{t} u^{k}, d_{t} u^{k}\right],
$$

where $G_{\varepsilon}^{\prime \prime}\left[\xi^{k} ; d_{t} u^{k}, d_{t} u^{k}\right]$ is a formal representation of the Taylor remainder term

$\mathcal{R}_{G_{\varepsilon}}\left[u^{k-1}, u^{k} ; d_{t} u^{k}, d_{t} u^{k}\right]=\int_{0}^{1}(1-s) G_{\varepsilon}^{\prime \prime}\left[u^{k-1}+s\left(u^{k}-u^{k-1}\right) ; d_{t} u^{k}, d_{t} u^{k}\right] \mathrm{d} s$.

With the bounds for $u^{k}$ and $u^{k-1}$ we obtain that

$$
\left|G_{\varepsilon}^{\prime \prime}\left[\xi^{k} ; d_{t} u^{k}, d_{t} u^{k}\right]\right| \leq c_{2}\left(1+\left\|\left[\xi^{k}\right]^{\prime \prime}\right\|\right)\left\|\left[d_{t} u^{k}\right]^{\prime \prime}\right\|^{2} \leq c_{2}^{\prime} \varepsilon^{-1}\left\|\left[d_{t} u^{k}\right]^{\prime \prime}\right\|^{2} .
$$

We thus obtain that

$$
\begin{aligned}
\left\|d_{t} u^{k}\right\|_{*}^{2}+d_{t} & \frac{1}{2}\left\|\left[u^{k}\right]^{\prime \prime}\right\|^{2}+\frac{1}{2}\left\|\left[d_{t} u^{k}\right]^{\prime \prime}\right\|^{2}=G_{\varepsilon}^{\prime}\left[u^{k-1} ; d_{t} u^{k}\right] \\
& =d_{t} G_{\varepsilon}\left[u^{k}\right]-\tau G_{\varepsilon}^{\prime \prime}\left[\xi^{k} ; d_{t} u^{k}, d_{t} u^{k}\right] \leq d_{t} G_{\varepsilon}\left[u^{k}\right]+\tau c_{2}^{\prime} \varepsilon^{-1}\left\|d_{t} u^{k}\right\|_{*}^{2} .
\end{aligned}
$$

This proves the energy monotonicity and hence part (i) of the proposition. Part (ii) follows as in the proof of Proposition 3.2 .

3.3. Conical sheet indentation flow. To iteratively solve the reduced conical sheet indentation problem $\left(\overline{\mathrm{P}_{\text {ind }}}\right)$ we include the obstacle condition $u_{s}(x) \geq \delta$ via a penalty term in the energy functional, i.e.,

$$
I_{\varepsilon}[u]=\frac{1}{2} \int_{S^{1}}\left|u^{\prime \prime}\right|^{2} \mathrm{~d} x+\frac{1}{2 \varepsilon} \int_{S^{1}}\left(u_{3}-\delta\right)_{-}^{2} \mathrm{~d} x,
$$

where $(s)_{-}=\min \{s, 0\}$. The discretization of the related gradient flow

$$
\left(\partial_{t} u, v\right)_{*}+\left(u^{\prime \prime}, v^{\prime \prime}\right)+\varepsilon^{-1}\left(\left(u_{3}-\delta\right)_{-}, v_{3}\right)=0
$$

uses the convex-concave splitting

$$
\left(u_{3}-\delta\right)_{-}^{2}=\left(u_{3}-\delta\right)^{2}-\left(u_{3}-\delta\right)_{+}^{2}
$$

and an implicit treatment of the corresponding monotone and an explicit treatment of the corresponding antimonotone terms, i.e., we use the timestepping scheme

$$
\left(d_{t} u^{k}, v\right)_{*}+\left(\left[u^{k}\right]^{\prime \prime}, v^{\prime \prime}\right)+\varepsilon^{-1}\left(u_{3}^{k}-\delta, v_{3}\right)=\varepsilon^{-1}\left(\left(u_{3}^{k-1}-\delta\right)_{+}, v_{3}\right) .
$$

A spatial discretization leads to the following algorithm where periodicity is guaranteed via an appropriate definition of the operator $L_{\mathrm{bc}}$.

Algorithm 3.5 (Conical sheet flow). Choose $u_{h}^{0} \in V_{h}$ such that $\mathcal{I}_{h} \Phi_{S}\left(u_{h}^{0}\right)=$ 0 and $\mathcal{I}_{h}\left|\left[u_{h}^{0}\right]^{\prime}\right|^{2}=1$ and $\mathcal{I}_{h} u_{h}^{0} \geq \delta$ and $L_{\mathrm{bc}}\left[u_{h}^{0}\right]=\ell_{\mathrm{bc}}$. Set $k=0$.

(1) Compute $d_{t} u_{h}^{k} \in V_{h}$ such that

$$
\begin{aligned}
\left(d_{t} u_{h}^{k}, v_{h}\right)_{*}+\left(\left[u_{h}^{k-1}+\tau d_{t} u_{h}^{k}\right]^{\prime \prime}, v_{h}^{\prime \prime}\right)+\varepsilon^{-1} & \left(u_{h, 3}^{k}-\delta, v_{h, 3}\right)_{h} \\
& =\varepsilon^{-1}\left(\left(u_{h, 3}^{k-1}-\delta\right)_{+}, v_{h, 3}\right)_{h}
\end{aligned}
$$


for all $v_{h} \in V_{h}$ subject to the constraints

$$
d_{t} u_{h}^{k}, v_{h} \in \mathcal{F}_{h}\left[u_{h}^{k-1}\right] .
$$

(2) Define $u_{h}^{k}=u_{h}^{k-1}+\tau d_{t} u_{h}^{k}$; set $k \rightarrow k+1$, and continue with (1).

The iteration of Algorithm 3.5 has the same features as that of Algorithm 3.1. We use the discrete penalized energy functional

$$
I_{h, \varepsilon}\left[u_{h}\right]=\frac{1}{2} \int_{S^{1}}\left|u_{h}^{\prime \prime}\right|^{2} \mathrm{~d} x+\frac{1}{2 \varepsilon} \int_{S^{1}} \mathcal{I}_{h}\left(u_{h, 3}-\delta\right)_{-}^{2} \mathrm{~d} x .
$$

Proposition 3.6. (i) Assume that $u_{h}^{0} \in \mathcal{A}_{h}$ with $u_{h, 3}^{0} \geq \delta$. Algorithm 3.5 defines a sequence $\left(u_{h}^{k}\right)_{k=0,1, \ldots} \subset V_{h}$ such that for every $K \geq 0$ we have

$$
I_{h, \varepsilon}\left[u_{h}^{K}\right]+\tau \sum_{k=1}^{K}\left\|d_{t} u_{h}^{k}\right\|_{*}^{2} \leq I_{h, \varepsilon}\left[u_{h}^{0}\right]=e_{0, h} .
$$

(ii) Under the above conditions the bounds on the constraint violation errors apply as in Proposition 3.2 (ii).

Proof. We follow the steps of the proof of Proposition 3.2 and use $v_{h}=d_{t} u_{h}^{k}$ in Step (1) of Algorithm 3.5. Defining the convex and concave functions $p_{c x}$ and $p_{c v}$, suitably embedded into $\mathbb{R}^{3}$, via

$$
p_{c x}(s)=\left(s_{3}-\delta\right)^{2} e_{3}, \quad p_{c v}(s)=-\left(s_{3}-\delta\right)_{+}^{2} e_{3},
$$

with the canonical basis vector $e_{3} \in \mathbb{R}^{3}$, we thus have

$$
\begin{aligned}
\left\|d_{t} u^{k}\right\|_{*}^{2}+d_{t} & \frac{1}{2}\left\|\left[u_{h}^{k}\right]^{\prime \prime}\right\|^{2}+\frac{\tau}{2}\left\|\left[d_{t} u_{h}^{k}\right]^{\prime \prime}\right\|^{2} \\
& =-\varepsilon^{-1}\left(p_{c x}^{\prime}\left(u_{h}^{k}\right), d_{t} u_{h}^{k}\right)-\varepsilon^{-1}\left(p_{c v}^{\prime}\left(u_{h}^{k-1}\right), d_{t} u_{h}^{k}\right) .
\end{aligned}
$$

The convexity of $p_{c x}$ and $-p_{c v}$ imply that we have

$$
\begin{array}{r}
p_{c x}^{\prime}\left(u_{h}^{k}\right) \cdot\left(u_{h}^{k-1}-u_{h}^{k}\right)+p_{c x}\left(u_{h}^{k}\right) \leq p_{c x}\left(u_{h}^{k-1}\right), \\
-p_{c v}^{\prime}\left(u_{h}^{k-1}\right) \cdot\left(u_{h}^{k}-u_{h}^{k-1}\right)-p_{c v}\left(u_{h}^{k-1}\right) \leq-p_{c v}\left(u_{h}^{k}\right) .
\end{array}
$$

By adding the inequalities and dividing by $\tau$ we find that

$$
-\left(p_{c x}^{\prime}\left(u_{h}^{k}\right)+p_{c v}^{\prime}\left(u_{h}^{k-1}\right)\right) \cdot d_{t} u_{h}^{k} \leq-d_{t}\left(p_{c x}\left(u_{h}^{k}\right)+p_{c v}\left(u_{h}^{k}\right)\right) .
$$

Combining the estimates implies the unconditional energy decay property. The remaining part (ii) is derived as in the proof of Proposition 3.2 .

Remark 3.7. To obtain a consistency property for the discrete gradient flow as an approximation of a corresponding continuous gradient flow a condition relating the step-size $\tau$ and the penalty parameter $\varepsilon$ is required. 


\section{NUMERICAL EXPERIMENTS}

We illustrate the performance of the numerical methods devised in the previous sections by various numerical experiments which are specified in the following subsections. The implementation of the algorithms was realized in MATLAB with a direct solution of the linear systems of equations. The evolution metric $(\cdot, \cdot)_{*}$ was always chosen to coincide with $L^{2}$ inner product which leads to a mesh-dependent constant $c_{*}$ in Propositions 3.2, 3.4, and 3.6. We observe however good stability properties for the resulting discrete $L^{2}$ flow.

4.1. Elastic and geodesic flows on a torus. We compare discrete relaxation dynamics for curves on a torus that are determined by the elastic bending energy and by the geodesic curvature functional. The torus $T_{r, R}$ has radii $R=2$ and $r=1$ and is described by the zero level set of the function

$$
\Phi_{S}(s)=\left(|s|^{2}+R^{2}-r^{2}\right)^{2}-4 R^{2}\left(|s|^{2}-s_{3}^{2}\right) .
$$

The following example defines an open curve on $T_{r, R}$.

Example 4.1. Let $\widetilde{L}=2 \pi$ and for $x \in(0, \widetilde{L})$ define $\widetilde{u}^{0}:(0, \widetilde{L}) \rightarrow T_{r, R}$ via

$$
\widetilde{u}^{0}(x)=\left[\begin{array}{c}
\sin (a x)(R+\sin (b x) r) \\
\cos (a x)(R+\sin (b x) r) \\
\cos (b x) r
\end{array}\right]
$$

The curve $u^{0}:(0, L) \rightarrow T_{r, R}$ is obtained from a re-parametrization of $\widetilde{u}^{0}$.

We use clamped boundary conditions at $x=0$ that fix the initial position and tangent, i.e., we have

$$
L_{\mathrm{bc}}[u]=\left(u(0), u^{\prime}(0)\right) .
$$

For a partition of the interval $(0, L)$ we ran Algorithms 3.1 and 3.3 with the parameters

$$
J=80, \quad h=2 \pi / J, \quad \tau=h, \quad \gamma=1-h .
$$

Figure 2 shows snapshots of the iterations. We observe that the curve changes quicker initially in the case of the bending energy and slightly slower for the geodesic curvature functional. This behavior is also seen in the energy plot shown in Figure 3 where we plotted the energies in dependence of the iteration numbers. In Figure 4 we illustrate for the evolution of closed curves on the torus the necessity of a stabilizing damping factor for the geodesic curvature flow. When no stabilization is used, i.e., in case that $\gamma=1$, then energy monotonicity fails and the discrete curves fail to belong to a small neighborhood of the given surface. 

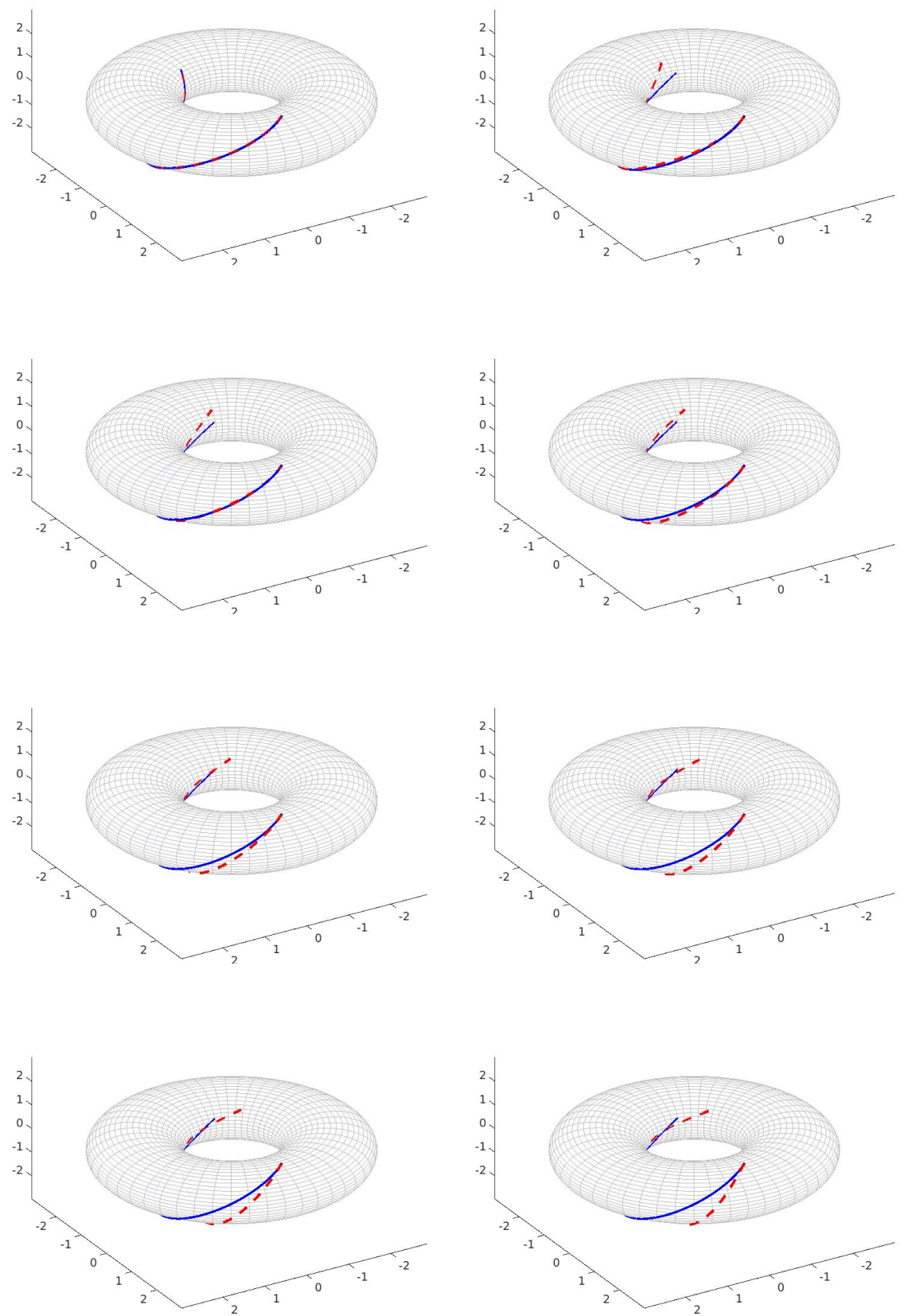

Figure 2. Snapshots of the discrete gradient flow evolutions after $n=0,20,40, \ldots, 160$ iterations for the bending (solid curves) and geodesic curvature (dashed curves) energies from the same initial curve. 


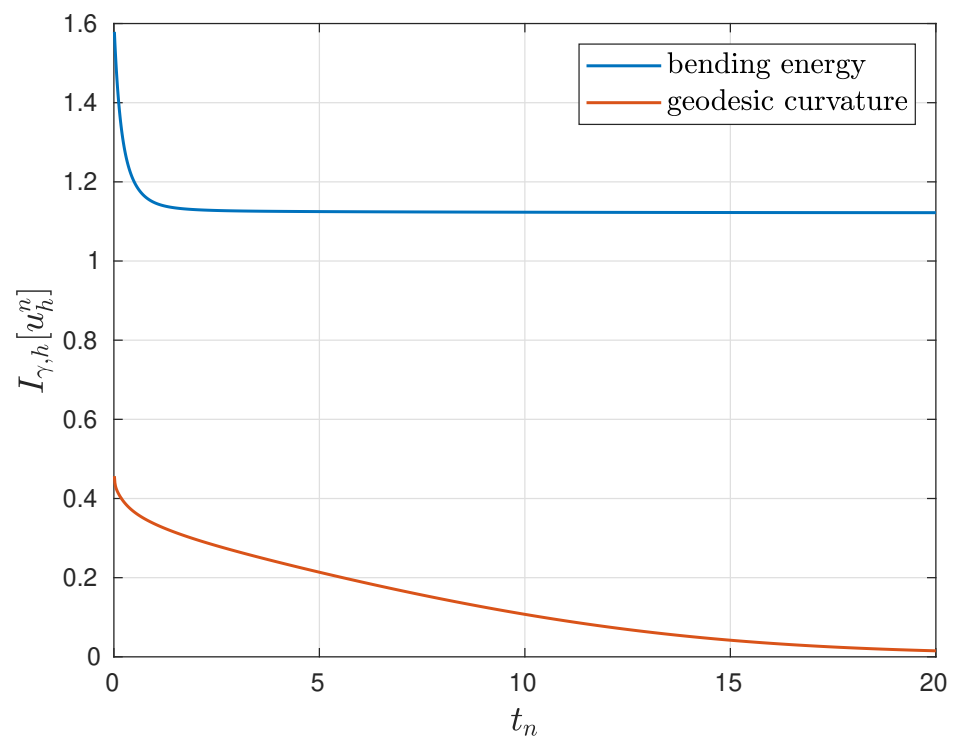

Figure 3. Decay of the bending energy and geodesic curvature functional for the evolution of clamped curves on a torus illustrated in Figure 2 .

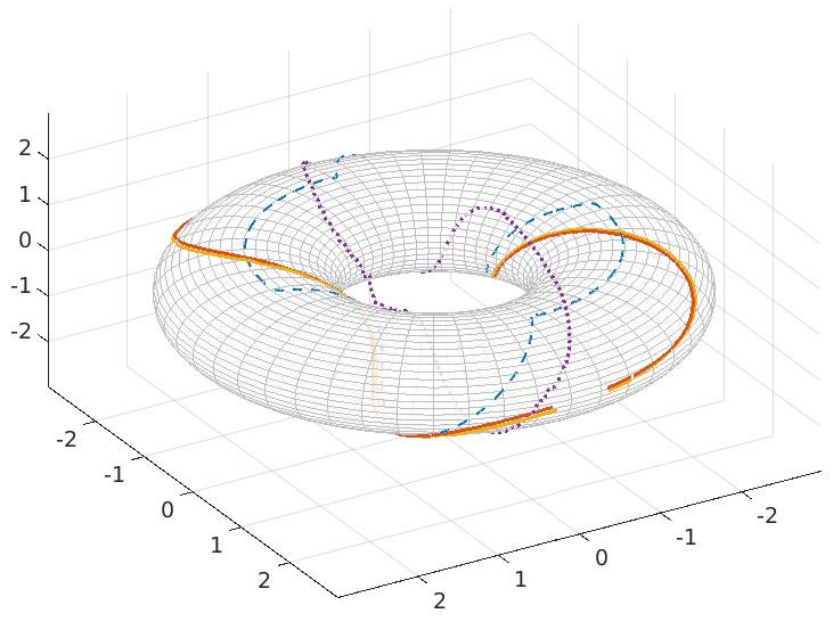

Figure 4. Initial closed curve (dashed) and corresponding relaxed curves for bending energy and geodesic curvature with stabilization (nearly coinciding solid curves) and configuration for geodesic curvature flow without stabilization (dotted irregular curve). 
4.2. Conical sheet indentation. We consider the following specification of the conical sheet indentation problem $\left(\mathrm{P}_{\text {ind }}\right)$.

Example 4.2 (Conical sheet). Let $\delta=1 / 4$ and $r=(15 / 16)^{2}$.

We used uniform partitions with mesh size $h>0$ and nodes $0=z_{0}<$ $\cdots<z_{J}=2 \pi$ of the cirle $S^{1}$ where $z_{0}$ and $z_{J}$ are identified in the sense that we impose the periodic boundary condition $L_{\mathrm{bc}}[u]=0$ with

$$
L_{\mathrm{bc}}[u]=\left(u\left(z_{J}\right)-u\left(z_{0}\right), u^{\prime}\left(z_{J}\right)-u^{\prime}\left(z_{0}\right)\right) .
$$

Figure 5 shows snapshots of the discrete evolution computed with Algorithm 3.5 for the discretization parameters

$$
J=80, \quad h=2 \pi / J, \quad \varepsilon=h^{2}, \quad \tau=h .
$$

The visualization displays the two-dimensional deformation of the elastic sheet by linearly connecting the origin with points on the curve. The initial configuration $u_{h}^{0}$ is a randomly generated function with corrected values to satisfy the condition $u_{h}^{0} \in \mathcal{A}_{h}$. The discrete evolution shows a rapid change to a smooth curve approximately obeying the obstacle constraint. In the following iterations the number of local maxima decreases until finally only one fold can be observed while the remaining part of the curve is in contact with the obstacle. Only a small penetration error occurs as can be seen in Figure 6, where we plotted the third component of the iterates $u_{h}^{n}$ with $n$ such that $t_{n}=n \tau=2$, i.e., $n=160$, for the choices

$$
\text { (i) } \varepsilon=h, \quad \text { (ii) } \varepsilon=h^{2}, \quad \text { (iii) } \varepsilon=h^{3} \text {. }
$$

For $\varepsilon=h$ we observe a strong penetration of the obstacle. Our energy monotonicity property implies the estimate

$$
\left\|\left(u_{3, h}^{n}-\delta\right)_{-}\right\| \leq\left(2 e_{0, h}\right)^{1 / 2} \varepsilon^{1 / 2}
$$

and from the experimental results we infer that $\varepsilon=h^{2}$ leads to the best results. It is also interesting to see how smaller penalization terms decrease the speed of the relaxation process. For $\varepsilon=h$ only one fold is present indicating stationarity, while for $\varepsilon=h^{2}$ and $\varepsilon=h^{3}$ a larger number of local maxima can be observed after 160 iteration steps. Figure 7 shows the decay of the bending energy for different resolutions and confirms the energy monotonicity and convergence to a stationary configuration. The large values of the energies are related to a strong dependence of minimal energies on the indentation depth $\delta$. For the significantly smaller choice $\delta=0.05$ we obtained the stationary energy values $I_{h, \varepsilon}\left[u_{h}^{n^{*}}\right]=24.104,17.828,21.822,21.569,21.565$ for discretizations with $J=40,80, \ldots, 640$ grid points. These values also confirms convergence of the discrete minimal energies as $h \rightarrow 0$.

Acknowledgments. The author is grateful to Rebecca Kromer for providing first versions of the numerical experiments. 

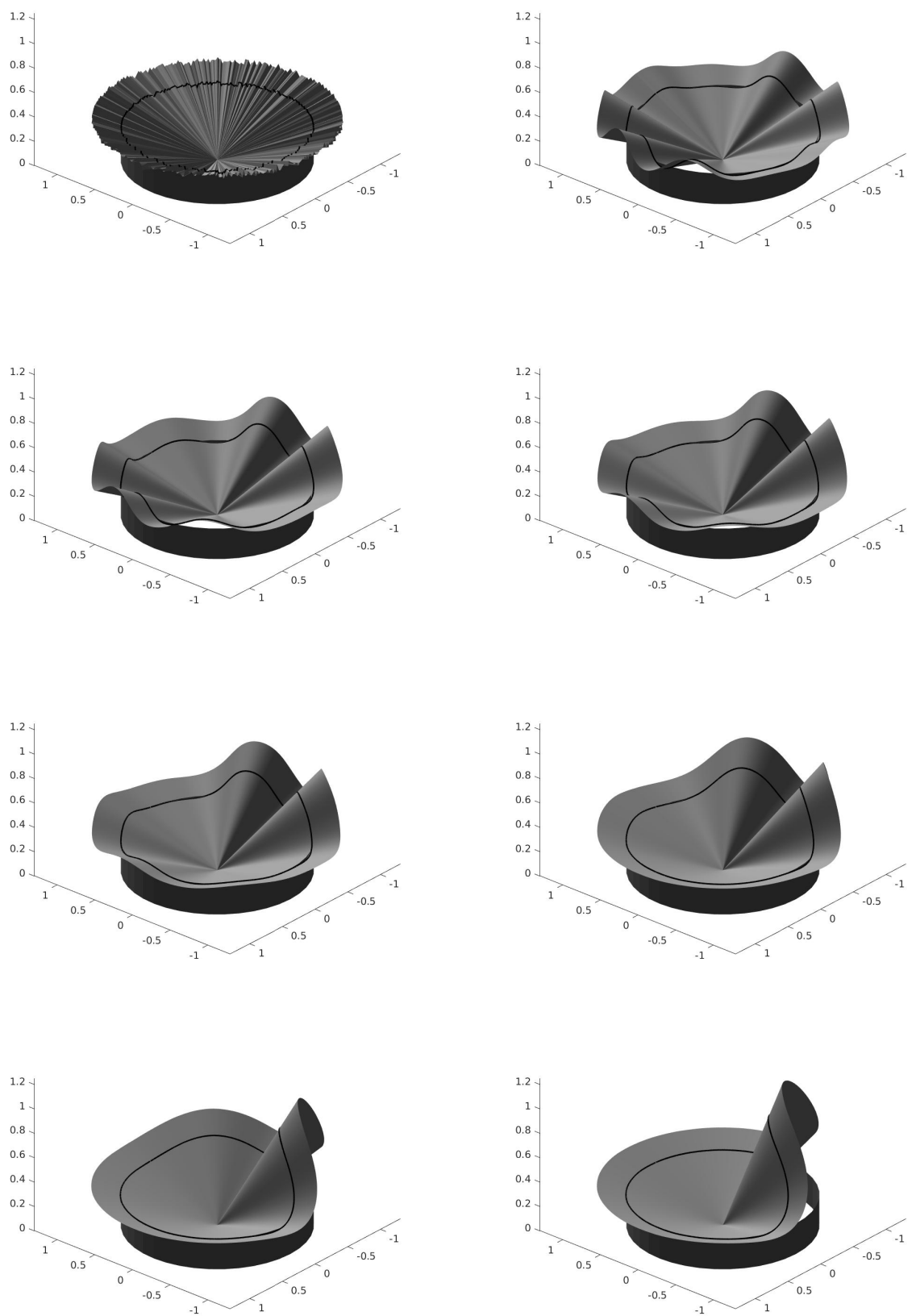

FIgURE 5. Snapshots of the discrete gradient flow for the sheet indentation problem after $n=$ $0,10,20,30,40,70,190,430$ iterations. The family of curves (solid lines) relax their bending energies until only one fold is present which is stationary and energy minimizing. 


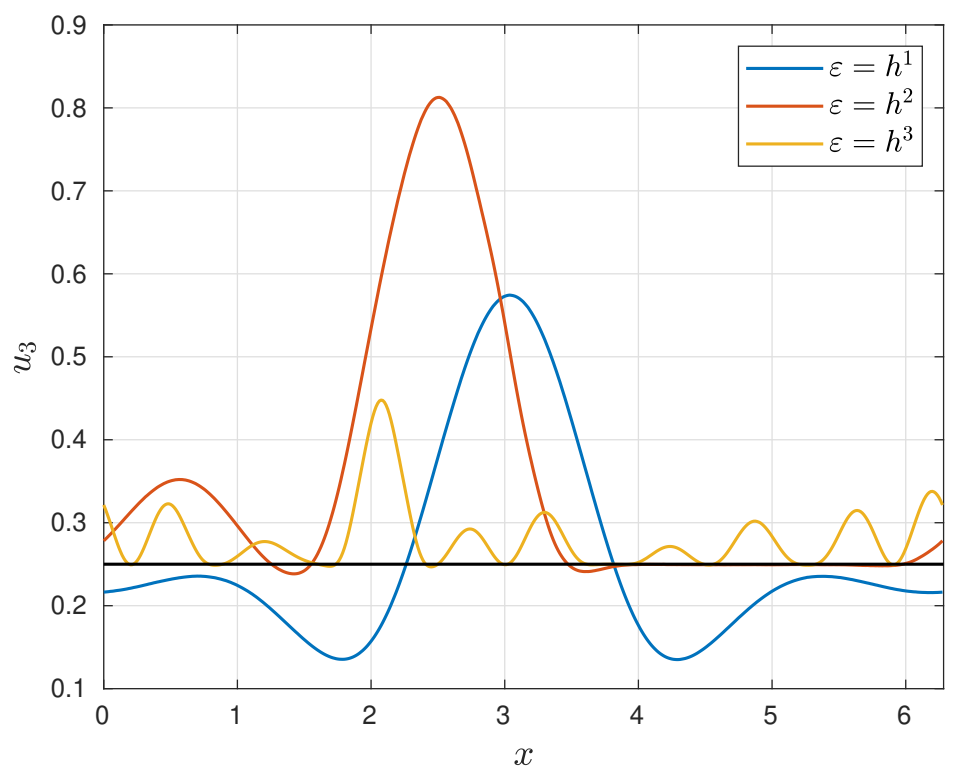

Figure 6 . Penetration of the obstacle at height $\delta=0.25$ (straight line) by the third component $u_{3, h}^{n}$ of the iterates in the sheet indentation problem after a fixed number $n=160$ of iterations for different choices of penalty parameters $\varepsilon$.

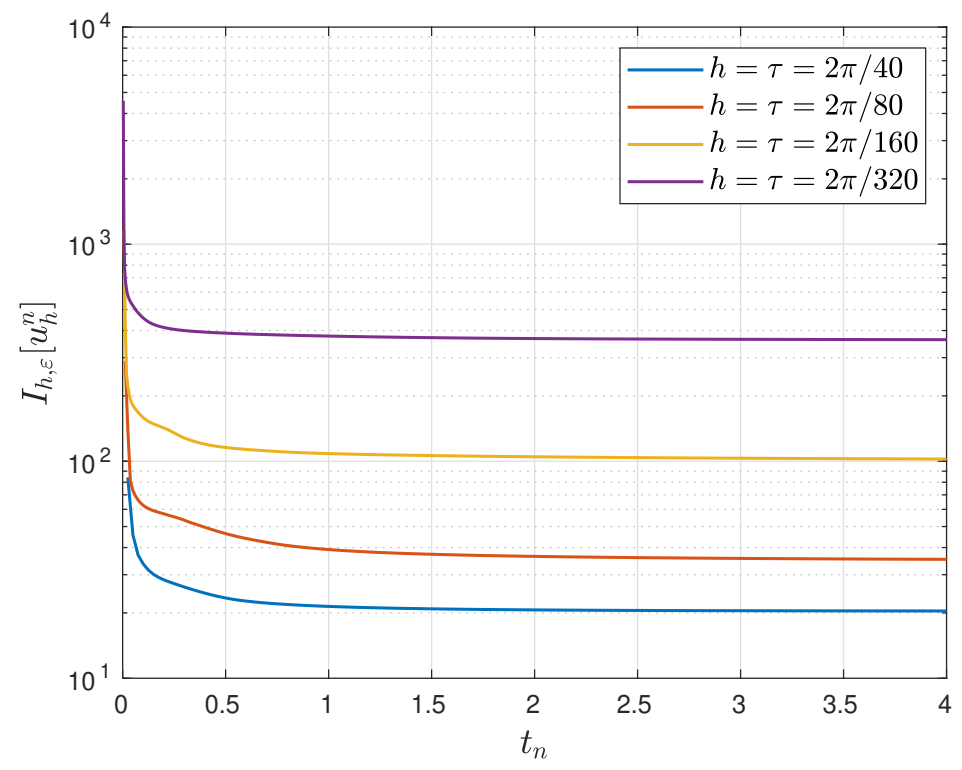

FIgURE 7. Energy decay $n \mapsto I_{h, \varepsilon}\left[u_{h}^{n}\right]$ in the sheet indentation problem for different spatial resolutions for meshdependent randomly generated initial configurations of large bending energy. 


\section{REFERENCES}

[Ant05] S. S. Antman. Nonlinear problems of elasticity. Second. Vol. 107. Applied Mathematical Sciences. Springer, New York, 2005, pp. xviii+831.

[AP10] B. Audoly and Y. Pomeau. Elasticity and geometry. From hair curls to the non-linear response of shells, With a foreword by John W. Hutchinson. Oxford University Press, Oxford, 2010, pp. x+586.

$[$ Bar +07$] \quad$ J. W. Barrett, S. Bartels, X. Feng, and A. Prohl. "A convergent and constraintpreserving finite element method for the $p$-harmonic flow into spheres". In: SIAM J. Numer. Anal. 45.3 (2007), pp. 905-927. DOI: 10.1137/050639429

[Bar05] S. Bartels. "Stability and convergence of finite-element approximation schemes for harmonic maps". In: SIAM J. Numer. Anal. 43.1 (2005), pp. 220-238. DOI: 10.1137/040606594

[Bar13] S. Bartels. "A simple scheme for the approximation of the elastic flow of inextensible curves". In: IMA J. Numer. Anal. 33.4 (2013), pp. 1115-1125. DOI: 10.1093/imanum/drs041.

[Bar16] S. Bartels. "Projection-free approximation of geometrically constrained partial differential equations". In: Math. Comp. 85.299 (2016), pp. 1033-1049. DOI: $10.1090 / \mathrm{mcom} / 3008$

[BGN08] J. W. Barrett, H. Garcke, and R. Nürnberg. "Numerical approximation of anisotropic geometric evolution equations in the plane". In: IMA J. Numer. Anal. 28.2 (2008), pp. 292-330. DOI: 10.1093/imanum/drm013.

[BGN10] J. W. Barrett, H. Garcke, and R. Nürnberg. "Numerical approximation of gradient flows for closed curves in $\mathbb{R}^{d "}$. In: IMA J. Numer. Anal. 30.1 (2010), pp. 4-60. DOI: 10.1093/imanum/drp005.

[BGN11] J. W. Barrett, H. Garcke, and R. Nürnberg. "The approximation of planar curve evolutions by stable fully implicit finite element schemes that equidistribute". In: Numer. Methods Partial Differential Equations 27.1 (2011), pp. 130. DOI: $10.1002 /$ num. 20637

[BGN12] J. W. Barrett, H. Garcke, and R. Nürnberg. "Parametric approximation of isotropic and anisotropic elastic flow for closed and open curves". In: Numer. Math. 120.3 (2012), pp. 489-542. DOI: 10.1007/s00211-011-0416-x.

[BGN19] J. W. Barrett, H. Garcke, and R. Nürnberg. "Stable discretizations of elastic flow in Riemannian manifolds". In: SIAM J. Numer. Anal. 57.4 (2019), pp. 1987-2018. DOI: 10.1137/18M1227111.

[BKN13] J. Brandman, R. V. Kohn, and H.-M. Nguyen. "Energy scaling laws for conically constrained thin elastic sheets". In: J. Elasticity 113.2 (2013), pp. 251264. DOI: 10.1007/s10659-012-9420-3.

[BR19] S. Bartels and P. Reiter. Numerical solution of a bending-torsion model for elastic rods. 2019. arXiv: 1911.07024 [math.NA]

[BRR18] S. Bartels, P. Reiter, and J. Riege. "A simple scheme for the approximation of self-avoiding inextensible curves". In: IMA J. Numer. Anal. 38.2 (2018), pp. 543-565. DOI: 10.1093/imanum/drx021.

[BS08] S. C. Brenner and L. R. Scott. The mathematical theory of finite element methods. Third. Vol. 15. Texts in Applied Mathematics. Springer, New York, 2008, pp. xviii+397. DOI: 10.1007/978-0-387-75934-0.

[CGM06] N. Chouaieb, A. Goriely, and J. H. Maddocks. "Helices". In: Proc. Natl. Acad. Sci. USA 103.25 (2006), pp. 9398-9403. DOI: 10.1073/pnas.0508370103

[CM05] E. Cerda and L. Mahadevan. "Confined developable elastic surfaces: cylinders, cones and the Elastica". In: Proc. R. Soc. Lond. Ser. A Math. Phys. Eng. Sci. 461.2055 (2005), pp. 671-700. DOI: 10.1098/rspa.2004.1371. 
[CS00] B. D. Coleman and D. Swigon. "Theory of supercoiled elastic rings with selfcontact and its application to DNA plasmids". In: J. Elasticity 60.3 (2000), 173-221 (2001). DOI: 10.1023/A :1010911113919.

[DD09] K. Deckelnick and G. Dziuk. "Error analysis for the elastic flow of parametrized curves". In: Math. Comp. 78.266 (2009), pp. 645-671.

[DDE05] K. Deckelnick, G. Dziuk, and C. M. Elliott. "Computation of geometric partial differential equations and mean curvature flow". In: Acta Numer. 14 (2005), pp. 139-232. DOI: 10.1017/S0962492904000224.

[DKS02] G. Dziuk, E. Kuwert, and R. Schätzle. "Evolution of elastic curves in $\mathbb{R}^{n}$ : existence and computation". In: SIAM J. Math. Anal. 33.5 (2002), pp. 12281245. DOI: $10.1137 /$ S0036141001383709.

[DLP14] A. Dall'Acqua, C.-C. Lin, and P. Pozzi. "Evolution of open elastic curves in $\mathbb{R}^{n}$ subject to fixed length and natural boundary conditions". In: Analysis (Berlin) 34.2 (2014), pp. 209-222. DOI: 10.1515/anly-2014-1249.

[FM18] A. Figalli and C. Mooney. "An obstacle problem for conical deformations of thin elastic sheets". In: Arch. Ration. Mech. Anal. 228.2 (2018), pp. 401-429. DOI: $10.1007 / \mathrm{s} 00205-017-1195-\mathrm{z}$

[Koi96] N. Koiso. "On the motion of a curve towards elastica". In: Actes de la Table Ronde de Géométrie Différentielle (Luminy, 1992). Vol. 1. Sémin. Congr. Soc. Math. France, Paris, 1996, pp. 403-436.

[Lin91] A. Linnér. "Curve-straightening in closed Euclidean submanifolds". In: Comm. Math. Phys. 138.1 (1991), pp. 33-49.

[LS85] J. Langer and D. A. Singer. "Curve straightening and a minimax argument for closed elastic curves". In: Topology 24.1 (1985), pp. 75-88. DOI: 10.1016/00409383(85)90046-1

[MM03] M. G. Mora and S. Müller. "Derivation of the nonlinear bending-torsion theory for inextensible rods by $\Gamma$-convergence". In: Calc. Var. Partial Differential Equations 18.3 (2003), pp. 287-305. DOI: $10.1007 /$ s00526-003-0204-2.

[MO14] S. Müller and H. Olbermann. "Conical singularities in thin elastic sheets". In: Calc. Var. Partial Differential Equations 49.3-4 (2014), pp. 1177-1186. DOI: 10.1007/s00526-013-0616-6

[Olb16] H. Olbermann. "The one-dimensional model for d-cones revisited". In: $A d v$. Calc. Var. 9.3 (2016), pp. 201-215. DOI: 10.1515/acv-2014-0031

[PS17] P. Pozzi and B. Stinner. "Curve shortening flow coupled to lateral diffusion". In: Numer. Math. 135.4 (2017), pp. 1171-1205. DOI: 10.1007/s00211-0160828-8. 